\title{
Block diagonalization using similarity renormalization group flow equations
}

\author{
E. Anderson, ${ }^{1}$ S. K. Bogner,${ }^{2}$ R. J. Furnstahl, ${ }^{1}$ E. D. Jurgenson, ${ }^{1}$ R. J. Perry, ${ }^{1}$ and A. Schwenk ${ }^{3}$ \\ ${ }^{1}$ Department of Physics, The Ohio State University, Columbus, Ohio 43210, USA \\ ${ }^{2}$ National Superconducting Cyclotron Laboratory and \\ Department of Physics and Astronomy, Michigan State University, East Lansing, Michigan 48844, USA \\ ${ }^{3}$ TRIUMF, 4004 Wesbrook Mall, Vancouver, British Columbia, Canada V6T 2A3 \\ (Received 14 January 2008; published 24 March 2008)
}

\begin{abstract}
By choosing appropriate generators for the Similarity Renormalization Group (SRG) flow equations, different patterns of decoupling in a Hamiltonian can be achieved. Sharp and smooth block-diagonal forms of phase-shift equivalent nucleon-nucleon potentials in momentum space are generated as examples and compared to analogous low-momentum interactions (" $V_{\text {low } k}$ ").
\end{abstract}

DOI: 10.1103/PhysRevC.77.037001

PACS number(s): 21.30.-x, 05.10.Cc, 13.75.Cs

The Similarity Renormalization Group (SRG) [1-3] applied to internucleon interactions is a continuous series of unitary transformations implemented as a flow equation for the evolving Hamiltonian $H_{s}$,

$$
\frac{d H_{s}}{d s}=\left[\eta_{s}, H_{s}\right]=\left[\left[G_{s}, H_{s}\right], H_{s}\right] .
$$

Here $s$ is a flow parameter and the flow operator $G_{s}$ specifies the type of SRG [4]. Decoupling between low-energy and highenergy matrix elements is naturally achieved in a momentum basis by choosing a momentum-diagonal flow operator such as the kinetic energy $T_{\text {rel }}$ or the diagonal of $H_{s}$; either drives the Hamiltonian toward band-diagonal form. This decoupling leads to dramatically improved variational convergence in fewbody nuclear systems compared to unevolved phenomenological or chiral effective field theory (EFT) potentials $[5,6]$.

Renormalization Group (RG) methods that evolve $N N$ interactions with a sharp or smooth cutoff in relative momentum, known generically as $V_{\text {low } k}$, rely on the invariance of the two-nucleon $\mathrm{T}$ matrix $[7,8]$. These approaches achieve a block-diagonal form characterized by a cutoff $\Lambda$ (see left plots in Figs. 1 and 2). As implemented in Refs. [7,8], the high-momentum matrix elements are defined to be zero, but this is not required.

Block-diagonal decoupling of the sharp $V_{\text {low } k}$ form can be generated using SRG flow equations by choosing a blockdiagonal flow operator (see Refs. $[9,10]$ for details),

$$
G_{s}=\left(\begin{array}{cc}
P H_{s} P & 0 \\
0 & Q H_{s} Q
\end{array}\right) \equiv H_{s}^{\mathrm{bd}},
$$

with projection operators $P$ and $Q=1-P$. In a partial-wave momentum representation, $P$ and $Q$ are step functions defined by a sharp cutoff $\Lambda$ on relative momenta. This choice for $G_{s}$, which means that $\eta_{s}$ is nonzero only where $G_{s}$ is zero, suppresses off-diagonal matrix elements such that the Hamiltonian approaches a block-diagonal form as $s$ increases. If one considers a measure of the off-diagonal coupling of the Hamiltonian,

$$
\operatorname{Tr}\left[\left(Q H_{s} P\right)^{\dagger}\left(Q H_{s} P\right)\right]=\operatorname{Tr}\left[P H_{s} Q H_{S} P\right] \geqslant 0,
$$

then its derivative is easily evaluated by applying the SRG equation, Eq. (1):

$$
\begin{aligned}
\frac{d}{d s} \operatorname{Tr}\left[P H_{s} Q H_{s} P\right] \\
=\operatorname{Tr}\left[P \eta_{s} Q\left(Q H_{s} Q H_{s} P-Q H_{s} P H_{s} P\right)\right] \\
\quad+\operatorname{Tr}\left[\left(P H_{s} P H_{s} Q-P H_{s} Q H_{s} Q\right) Q \eta_{s} P\right] \\
=-2 \operatorname{Tr}\left[\left(Q \eta_{s} P\right)^{\dagger}\left(Q \eta_{s} P\right)\right] \leqslant 0 .
\end{aligned}
$$

Thus, the off-diagonal $Q H_{s} P$ block will decrease in general as $s$ increases $[9,10]$.

The right plots in Figs. 1 and 2 result from evolving the $\mathrm{N}^{3} \mathrm{LO}$ potential from Ref. [11] using the block-diagonal $G_{s}$ of Eq. (2) with $\Lambda=2 \mathrm{fm}^{-1}$ until $\lambda \equiv 1 / s^{1 / 4}=0.5 \mathrm{fm}^{-1}$. The parameter $\lambda$ is a quantitative measure of the progress toward block diagonalization made by the SRG evolution. The agreement between $V_{\text {low } k}$ and SRG potentials for momenta below $\Lambda$ is striking. A similar degree of universality is found in the other partial waves. Deriving an explicit connection between these approaches is the topic of an ongoing investigation.

The evolution with $\lambda$ of two representative partial waves $\left({ }^{3} \mathrm{~S}_{1}\right.$ and $\left.{ }^{1} \mathrm{P}_{1}\right)$ are shown in Figs. 3 and 4 . The evolution of the "off-diagonal" matrix elements (meaning those outside the $P H_{S} P$ and $Q H_{s} Q$ blocks) can be roughly understood from the dominance of the kinetic energy on the diagonal. Let the indices $p$ and $q$ run over indices of the momentum states in the $P$ and $Q$ spaces, respectively. To good approximation we can replace $P H_{s} P$ and $Q H_{s} Q$ by their eigenvalues $E_{p}$ and $E_{q}$ in the SRG equations, yielding $[9,10]$

$$
\frac{d}{d s} h_{p q} \approx \eta_{p q} E_{q}-E_{p} \eta_{p q}=-\left(E_{p}-E_{q}\right) \eta_{p q}
$$

and

$$
\eta_{p q} \approx E_{p} h_{p q}-h_{p q} E_{q}=\left(E_{p}-E_{q}\right) h_{p q} .
$$

Combining these two results, we have the evolution of any off-diagonal matrix element:

$$
\frac{d}{d s} h_{p q} \approx-\left(E_{p}-E_{q}\right)^{2} h_{p q} .
$$




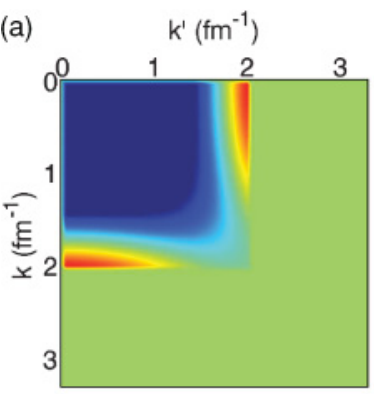

(b) $\quad \mathrm{k}^{\prime}\left(\mathrm{fm}^{-1}\right)$

FIG. 1. (Color online) Comparison of momentum-space (a) $V_{\text {low } k}$ and (b) SRG block-diagonal potentials with $\Lambda=2 \mathrm{fm}^{-1}$ evolved from an $\mathrm{N}^{3} \mathrm{LO}^{3} \mathrm{~S}_{1}$ potential [11].

(a)
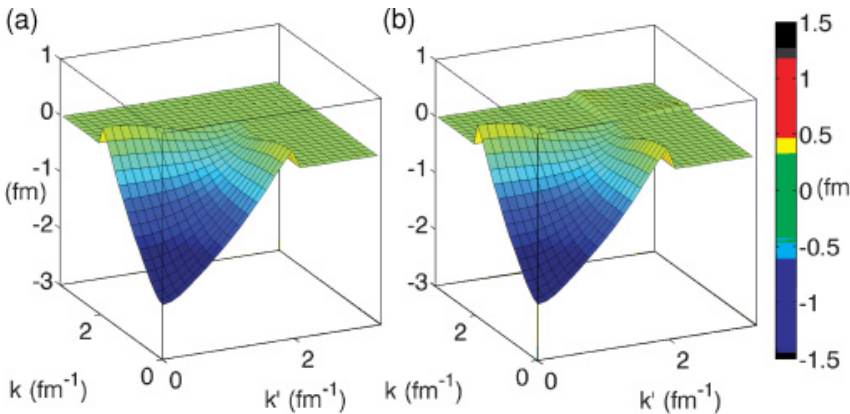

FIG. 2. (Color online) Comparison of momentum-space (a) $V_{\text {low } k}$ and (b) SRG block-diagonal potentials with $\Lambda=2 \mathrm{fm}^{-1}$ evolved from an $\mathrm{N}^{3} \mathrm{LO}^{3} \mathrm{~S}_{1}$ potential [11].
In the $N N$ case we can replace the eigenvalues by those for the relative kinetic energy, giving an explicit solution

$$
h_{p q}(s) \approx h_{p q}(0) e^{-s\left(\epsilon_{p}-\epsilon_{q}\right)^{2}}
$$

with $\epsilon_{p} \equiv p^{2} / M$. Thus the off-diagonal elements go to zero with the energy differences just like with the SRG with $T_{\text {rel }}$; one can see the width of order $1 / \sqrt{s}=\lambda^{2}$ in the $k^{2}$ plots of the evolving potential in Figs. 3 and 4.

While in principle the evolution to a sharp block-diagonal form means going to $s=\infty(\lambda=0)$, in practice we need only take $s$ as large as needed to quantitatively achieve the decoupling implied by Eq. (8). Furthermore, it should hold for more general definitions of $P$ and $Q$. To smooth out the cutoff, we can introduce a smooth regulator $f_{\Lambda}$, which we take here to be an exponential form:

$$
f_{\Lambda}(k)=e^{-\left(k^{2} / \Lambda^{2}\right)^{n}},
$$

with $n$ an integer. For $V_{\text {low } k}$ potentials, typical values used are $n=4$ and $n=8$ (the latter is considerably sharper but still numerically robust). By replacing $H_{s}^{\text {bd }}$ with

$$
G_{s}=f_{\Lambda} H_{s} f_{\Lambda}+\left(1-f_{\Lambda}\right) H_{s}\left(1-f_{\Lambda}\right),
$$

we get a smooth block-diagonal potential.

A representative example with $\Lambda=2 \mathrm{fm}^{-1}$ and $n=4$ is shown in Fig. 5. We can evolve to $\lambda=1.5 \mathrm{fm}^{-1}$ without a problem. For smaller $\lambda$ the overlap of the $P$ and $Q$ spaces becomes significant and the potential becomes distorted. This distortion indicates that there is no further benefit to evolving

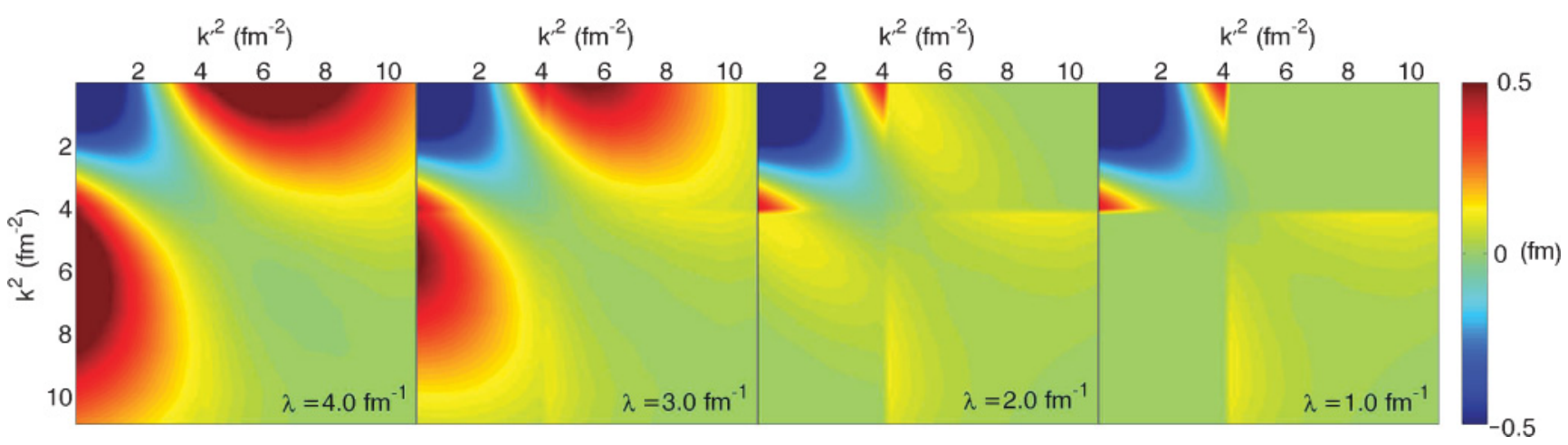

FIG. 3. (Color online) Evolution of the ${ }^{3} S_{1}$ partial wave with a sharp block-diagonal flow equation with $\Lambda=2 \mathrm{fm}^{-1}$ at $\lambda=4,3,2$, and $1 \mathrm{fm}^{-1}$. The initial $\mathrm{N}^{3} \mathrm{LO}$ potential is from Ref. [11].

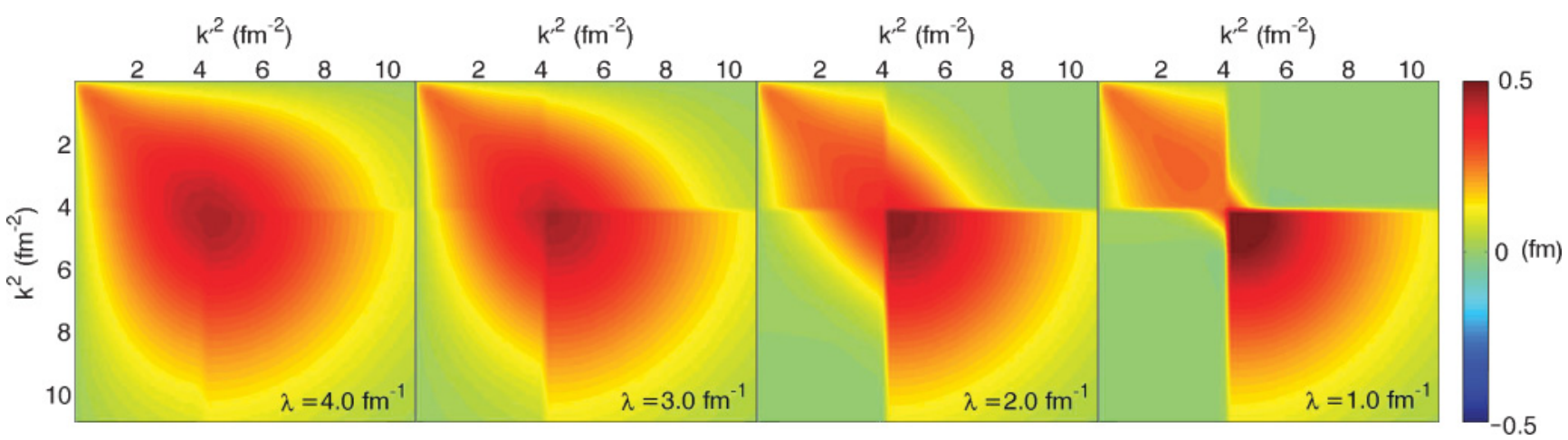

FIG. 4. (Color online) Same as Fig. 3 but for the ${ }^{1} \mathrm{P}_{1}$ partial wave. 


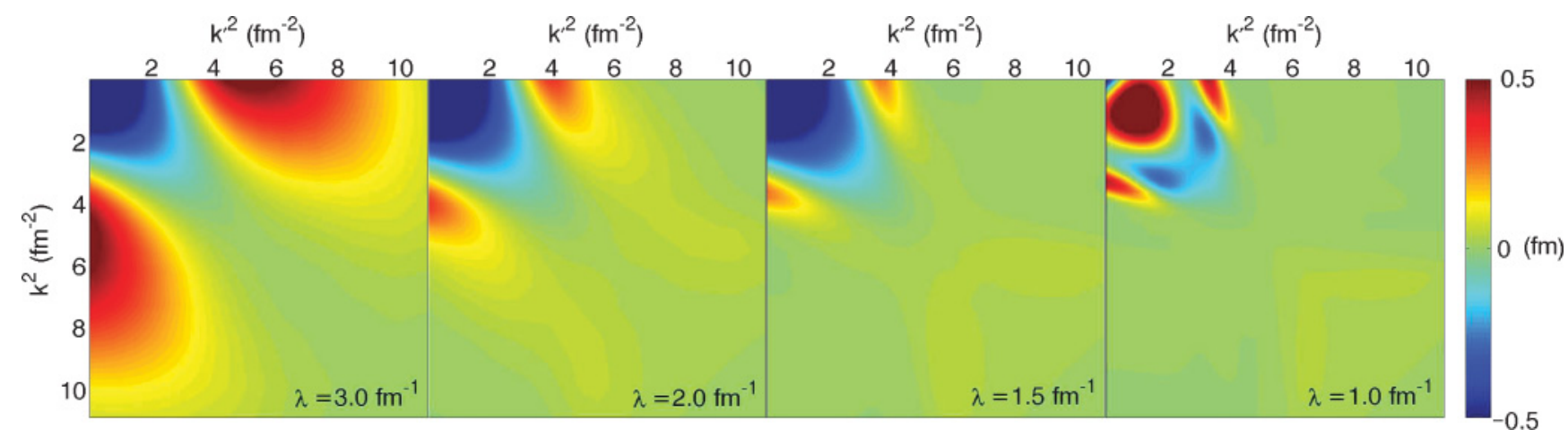

FIG. 5. (Color online) Evolution of the ${ }^{3} \mathrm{~S}_{1}$ partial wave with a smooth $(n=4)$ block-diagonal flow equation with $\Lambda=2.0 \mathrm{fm}{ }^{-1}$, starting with the $\mathrm{N}^{3} \mathrm{LO}$ potential from Ref. [11]. The flow parameter $\lambda$ is $3,2,1.5$, and $1 \mathrm{fm}^{-1}$.

in $\lambda$ very far below $\Lambda$; in fact the decoupling worsens for $\lambda<\Lambda$ with a smooth regulator.

Another type of SRG that is second-order exact and yields similar block diagonalization is defined by

$$
\eta_{s}=\left[T, P V_{s} Q+Q V_{s} P\right],
$$

which can be implemented with $P \rightarrow f_{\Lambda}$ and $Q \rightarrow\left(1-f_{\Lambda}\right)$, with $f_{\Lambda}$ either sharp or smooth. We can also consider bizarre choices for $f_{\Lambda}$ in Eq. (10), such as defining it to be zero out to $\Lambda_{\text {lower }}$, then unity out to $\Lambda$, and then zero above that. This means that $1-f_{\Lambda}$ defines both low and high-momentum blocks and the region that is driven to zero consists of several rectangles. Results for two partial waves starting from the Argonne $v_{18}$ potential [12] are shown in Fig. 6. Despite

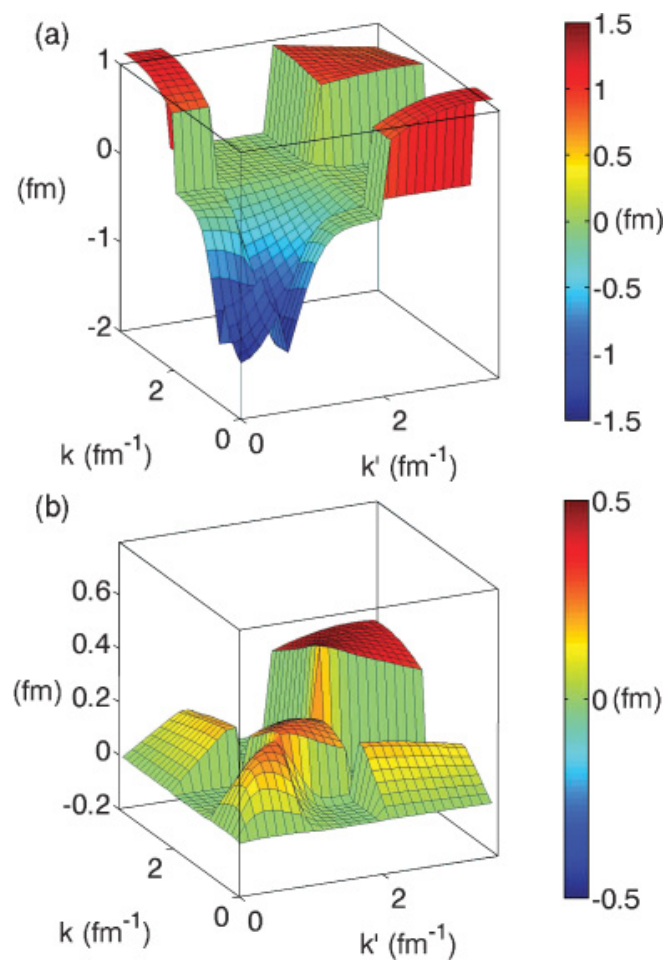

FIG. 6. (Color online) Evolved SRG potentials starting from Argonne $v_{18}$ in the (a) ${ }^{1} \mathrm{~S}_{0}$ and (b) ${ }^{1} \mathrm{P}_{1}$ partial waves to $\lambda=1 \mathrm{fm}^{-1}$ using a bizarre choice for $G_{s}$ (see text).

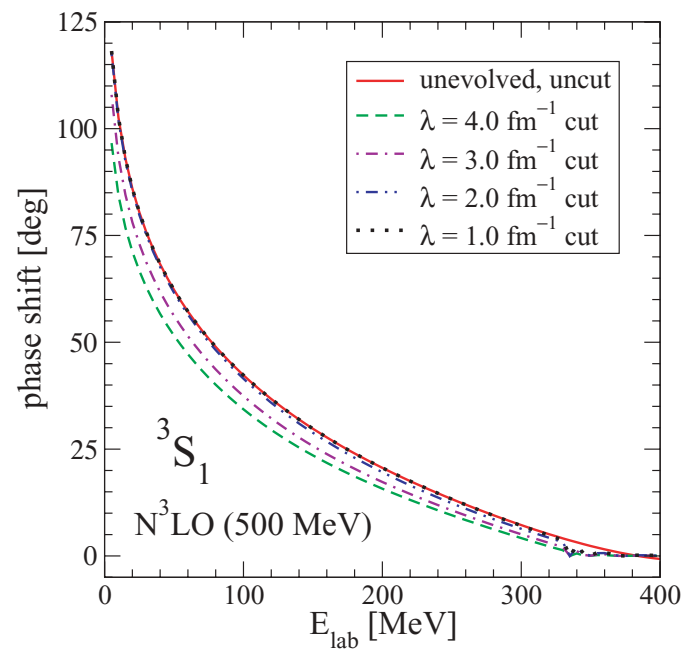

FIG. 7. (Color online) Phase shifts for the ${ }^{3} S_{1}$ partial wave from an initial $\mathrm{N}^{3} \mathrm{LO}$ potential and the evolved sharp SRG block-diagonal potential with $\Lambda=2 \mathrm{fm}^{-1}$ at various $\lambda$, in each case with the potential set identically to zero above $\Lambda$.

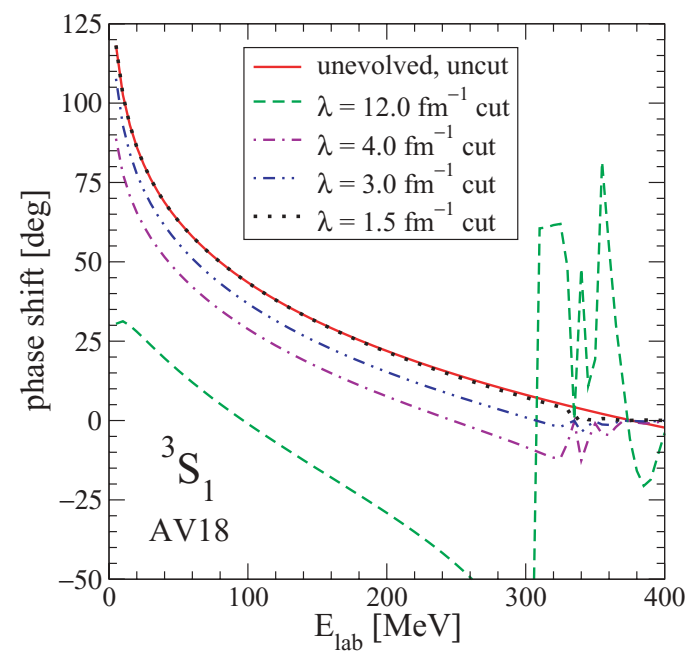

FIG. 8. (Color online) Same as Fig. 7 but with Argonne $v_{18}$ as the initial potential [12]. 


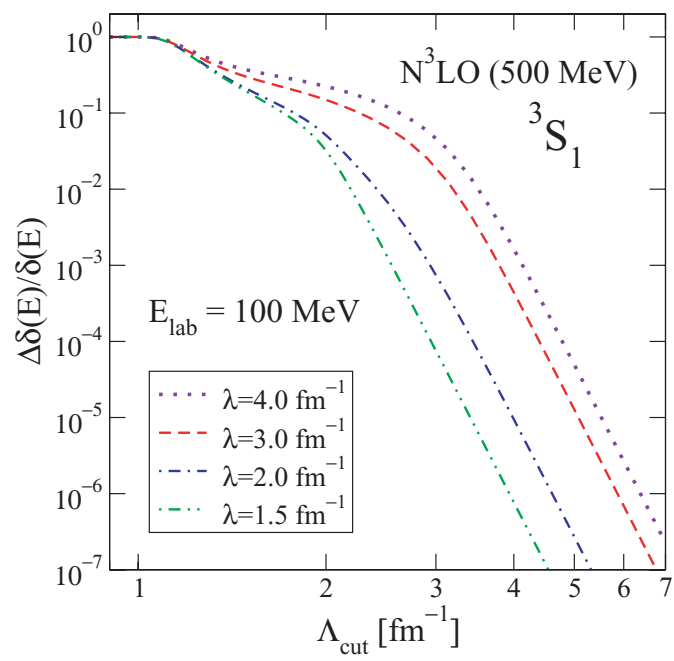

FIG. 9. (Color online) Errors in the phase shift at $E_{\text {lab }}=100 \mathrm{MeV}$ for the evolved sharp SRG block-diagonal potential with $\Lambda=2 \mathrm{fm}^{-1}$ for a range of $\lambda$ 's and a regulator with $n=8$.

the strange appearance, these remain unitary transformations of the original potential, with phase shifts and other $N N$ observables the same as with the original potential. These choices provide a proof-of-principle that the decoupled regions can be tailored to the physics problem at hand.

Definitive tests of decoupling for $N N$ observables are now possible for $V_{\text {low } k}$ potentials since the unitary transformation of the SRG guarantees that no physics is lost. For example, in Figs. 7 and 8 we show ${ }^{3} S_{1}$ phase shifts from an SRG sharp block diagonalization with $\Lambda=2 \mathrm{fm}^{-1}$ for two different potentials. The phase shifts are calculated with the potentials cut sharply at $\Lambda$. That is, the matrix elements of the potential are set to zero above that point. The improved decoupling as $\lambda$ decreases is evident in each case. By $\lambda=1 \mathrm{fm}^{-1}$ in Fig. 7 , the unevolved and evolved curves are indistinguishable to the width of the line up to about $300 \mathrm{MeV}$.

In Fig. 9 we show a quantitative analysis of the decoupling as in Ref. [13]. The figure shows the relative error of the phase shift at $100 \mathrm{MeV}$ calculated with a potential that is cut off by a smooth regulator as in Eq. (9) at a series of values $\Lambda_{\text {cut }}$. We observe the same universal decoupling behavior seen in Ref. [13]: a shoulder indicating the perturbative decoupling region, where the slope matches the power $2 n$ fixed by the smooth regulator. The onset of the shoulder in $\Lambda_{\text {cut }}$ decreases with $\lambda$ until it saturates for $\lambda$ somewhat below $\Lambda$, leaving the shoulder at $\Lambda_{\text {cut }} \approx \Lambda$. Thus, as $\lambda \rightarrow 0$ the decoupling scale is set by the cutoff $\Lambda$.

In the more conventional SRG, where we use $\eta_{s}=$ $\left[T, H_{s}\right]=\left[T, V_{s}\right]$, it is easy to see that the evolution of the twobody potential in the two-particle system can be carried over directly to the three-particle system. In particular, it follows that the three-body potential does not depend on disconnected two-body parts $[4,14]$. If we could implement $\eta_{s}$ as proposed here with analogous properties, we would have a tractable method for generating $V_{\text {low } k}$ three-body forces. While it seems possible to define Fock-space operators with projectors $P$ and $Q$ that will not have problems with disconnected parts, it is not yet clear whether full decoupling in the few-body space can be realized. Work on this problem is in progress.

This work was supported in part by the National Science Foundation under Grant Nos. PHY-0354916 and PHY0653312, the UNEDF SciDAC Collaboration under DOE Grant DE-FC02-07ER41457, and the Natural Sciences and Engineering Research Council of Canada (NSERC). TRIUMF receives federal funding via a contribution agreement through the National Research Council of Canada.
[1] S. D. Glazek and K. G. Wilson, Phys. Rev. D 48, 5863 (1993); 49, 4214 (1994).

[2] F. Wegner, Ann. Phys. (Leipzig) 3, 77 (1994); Phys. Rep. 348, 77 (2001).

[3] S. Kehrein, The Flow Equation Approach to Many-Particle Systems (Springer, Berlin, 2006).

[4] S. K. Bogner, R. J. Furnstahl, and R. J. Perry, Phys. Rev. C 75, 061001(R) (2007).

[5] S. K. Bogner, R. J. Furnstahl, R. J. Perry, and A. Schwenk, Phys. Lett. B649, 488 (2007).

[6] S. K. Bogner, R. J. Furnstahl, P. Maris, R. J. Perry, A. Schwenk, and J. P. Vary, Nucl. Phys. A801, 21 (2008).

[7] S. K. Bogner, T. T. S. Kuo, and A. Schwenk, Phys. Rep. 386, 1 (2003).
[8] S. K. Bogner, R. J. Furnstahl, S. Ramanan, and A. Schwenk, Nucl. Phys. A784, 79 (2007).

[9] E. L. Gubankova, H.-C. Pauli, F. J. Wegner, and G. Papp, arXiv:hep-th/9809143.

[10] E. L. Gubankova, C. R. Ji, and S. R. Cotanch, Phys. Rev. D 62, 074001 (2000).

[11] D. R. Entem and R. Machleidt, Phys. Rev. C 68, 041001(R) (2003).

[12] R. B. Wiringa, V. G. J. Stoks, and R. Schiavilla, Phys. Rev. C 51, 38 (1995).

[13] E. D. Jurgenson, S. K. Bogner, R. J. Furnstahl, and R. J. Perry, arXiv:0711.4266 [nucl-th].

[14] S. K. Bogner, R. J. Furnstahl, and R. J. Perry, arXiv:0708.1602 [nucl-th]. 